\title{
FURTHER PROPERTIES OF BORNOLOGICAL GROUPS
}

\author{
ANWAR N. IMRAN, I. S. RAKHIMOV
}

\begin{abstract}
In this work, Further properties of bornological groups are studied to find the sufficient conditions to introduce a bornology on a group. In particular, we show that every left (right) translations in bornological groups are isomorphisms and therefore the bornological group's structures are homogeneous, and this property from the bornological group is not shared with bornological semigroups. Further, the boundedness of a bornological group can be deduced from its boundedness at the identity.
\end{abstract}

Key words: bornological set; bornological group

\section{INTRODUCTION}

A bornological structure is very useful to solve the problem of boundedness of set and function, that means to determine the limits of the area. The idea of the bornological group (BG) came from starting to solve the problem of boundedness of the group instead of a set of elements. BG is set with two structure group and bornology such that the product map and inverse map are bounded. Note that every group can be turned into a bornological group by providing it with the discrete bornology. However, the problem of existence of non-discrete bornologies on infinite groups which would make them into bornological groups. This problem was solved by [4] in 2015, by introducing new structure bornological semigroups (BSG). BSG can be easily dene by taken semigroup instead of the group and require the product map is bounded, the condition of a bornological semigroup is weaker than the condition of a bornological group, so the problem of boundedness for this kind of groups, which cannot bornologies because the inverse map is not bounded was solved. But here, in this paper, we deal with the problem to understand what the sufficient condition should be imposed on bornological set to bornologies any group by studying further properties of bornological group and the motivation to study this kind of properties is that every left (right) translation to be an isomorphism and therefore the bornological group structure is homogeneous. Thus, we solve the problem of which bornological condition on bornological set can bornologies any group. As well as, that left (right) translation is isomorphism just in bornological group. So, this structure is totally different from bornological semigroup (see Example 3.5). For more detail in bornological group we refer to ([1], [2], [5], [6], [7]).

\section{Bornological Groups and Bornological Semigroups}

In this section, we present some background material on bornological groups (BG) and bornological semigroups (BSG), the reader can consult ([7], [4]).

Definition 2.1. A bornological group is a set $G$ with two structures:

(1) $G$ is group;

(2) $G$ is a bornological set, such that these two structures are compatible as follows:

(i) the product function $f_{1}: G \times G \longrightarrow G$ is bounded.

(ii) the inverse function $f_{2}: G \longrightarrow G$ is bounded. 


\section{Example 2.2.}

(i) Let $(N,\|\cdot\|)$ be a normed space. Define the bornology on $N$ as family of bounded subsets with respect to the norm. It is easy to see that $(N,\|\cdot\|, \beta)$ is a bornological group.

(ii) On $\mathbb{Q}$ each valuation, archimedean $|\cdot|_{\infty}$ or non-archimedean $|\cdot|_{p}$, give rise to a bornology on the additive group $(\mathbb{Q},+)$. A basis for the bornology is the set of closed disks

$$
D_{|\cdot|_{*}}\left(0, q^{+}\right)=\left\{x \in \mathbb{Q}:|x|_{*} \leq q\right\},
$$

and $q$ is a positive rational number with $* \in\{\infty, 2,3,5, \ldots\}$

Let $G$ be a bornological group and $A, B$ any two bounded subsets of $G$, we denote the image of $A \times B$ under the product map $G \times G \longrightarrow G$ by $A B=\{a b: a \in A, b \in B\}$. Similarly, $A^{-1}=\left\{a^{-1}: a \in A\right\}$ is the image of $A$ under the inverse map.

For $g \in G$ there is the right translation map $r_{g}: G \longrightarrow G$ defined by $r_{g}(x)=x \cdot g$ and the bijection map $l_{g}: G \longrightarrow G$ defined $l_{g}(x)=g x$ is called left translation by $g$.

Example 2.3. The multiplicative group $\left(\mathbb{C}^{*}, \cdot\right)$, endowed with the bornology induced by the usual bornology on $\mathbb{C}^{*}$ it is not bornological group since the inverse operation $x \longrightarrow x^{-1}$ is not bounded.

In the example, the second condition is not bounded, so we have a problem with this inverse function. So, we have to check if we delete this inverse function it will be a semigroup. That's why when we delete this inverse operation, this example and others come in. So, we give the concept of bornological semigroup. See the survey paper [4] for some results and for other reference.

Definition 2.4. A bornological semigroup is a nonempty set $S$ with two structures:

(1) $S$ is a semigroup with a binary operation $f: S \times S \longrightarrow S$;

(2) $S$ is a bornological set.

Such that the function $f$ is bounded.

Example 2.5. Let us consider the semigroup $(\mathbb{N},+)$ and $\beta$ be the family of all finite subsets of $\mathbb{N}$. Then $\mathbb{N}$ is a bornological semigroup.

Note that the above example is bornological semigroup but not bornological group. The group with discrete bornology is both a bornological groups and bornological semigroups.

Example 2.6. $\left(S_{3}, \circ, \beta\right)$ is both $B S G$ and $B G$.

Definition 2.7. Let $(X, \beta)$ and $\left(Y, \beta^{\prime}\right)$ be two bornological sets, a map $f: X \longrightarrow Y$ is called isomorphism if it is bijective, and $f, f^{-1}$ are bounded maps.

\section{Further Properties of Bornological Groups}

As we mention earlier a bornological group is a set with to structure group and bornology such that the product map $f: G \times G \longrightarrow G$ and the inverse map $h: G \longrightarrow G$ are bounded. 
In this section, an equivalent statement in terms of bounded set will now be presented.

Definition 3.1. A group $(G, *)$ with bornology $\beta$ is said to be a bornological group if for each $g_{1}, g_{2} \in G$ and each bounded sets $B_{1}, B_{2}$ containing $g_{1}, g_{2}$ respectively, there is $a$ bounded set $B$ contains $g_{1} * g_{2}^{-1}$ in $G$ such that $B_{1} * B_{2}^{-1} \subset B$

Proposition 3.2. Let $G$ be a bornological group. The following maps are isomorphism from $G$ to $G$ for all $g \in G$

(1) Every left translation $l_{g}$ and right translation $r_{g}$ in bornological group are isomorphisms

(2) The inverse map $x \longrightarrow x^{-1}$

(3) The inner automorphism map $x \longrightarrow g x g^{-1}$

Proof.

It is clear that, every left translation $l_{g}$ is a bijection. For $g \in G$ there is the left translation map $l_{g}: G \longrightarrow G$ defined by $l_{g}(h)=g h$. Clearly $l_{g h}=l_{g} \circ l_{h}$ and $l_{g^{-1}}=\left(l_{g}\right)^{-1}$. Since

$$
\left(l_{g} \circ l_{g^{-1}}\right)(x)=l_{g}\left(l_{g^{-1}}(x)\right)=l_{g}\left(g^{-1}(x)\right)=g g^{-1}(x)=l_{e}(x)=x=I d_{G},
$$

which it is the identity mapping, it follows that the inverse of $l_{g}$ is also bounded, that is, $l_{g}$ is an isomorphism of $G$ onto itself. A similar argument applies in the case of right translation of $G$. For the inverse mapping: we know it is a bijection since $G$ is a group (every element has a unique inverse), it is bounded by definition of a bornological group. The inverse map of the inverse map is it self, so it has a bounded inverse. Thus the inverse map is an isomorphism. finally, we have the automorphism map. This is a composition of two isomorphisms, $x \longrightarrow a x$ and $x \longrightarrow x a^{-1}$, and is thus an isomorphism.

An immediate and useful result we get from this proposition is as follows.

Corollary 3.3. Let $G$ be a bornological group and $g_{1}, g_{2} \in G$, There exists an isomorphism $f$ such that $f\left(g_{1}\right)=g_{2}$.

Proof. From Proposition 3.2 we know that $l_{g}$ is an isomorphism for all $g \in G$. by letting $f=r_{g_{1}^{-1} g_{2}}$ we get $f\left(g_{1}\right)=g_{2}$ as required.

By Proposition 3.2, we find that in every bornological group $G$ all right and left, translations are isomorphisms of $G$ onto itself.

However, a bornological set $X$ is said to be homogeneous if, for each $x, y \in X$, there exists an isomorphism $f$ of the space $X$ onto itself such that $f(x)=y$.

From Proposition 3.2, we obtain immediately, that bornological group structure is homogeneous space.

Theorem 3.4. Every bornological group is a homogeneous space.

Proof. Take any elements $a$ and $b$ in $G$, and put $c=b a^{-1}$. Then $\lambda_{c}(a)=c a=b a^{-1} a=b$. Since, by Proposition 3.2, $\lambda_{c}$ is an isomorphism which implies that the space $G$ is homogenous. 
Given a group $G$, it follows from Theorem 3.4 that to make $G$ into bornological group, we can only use homogenous. So, one of the main features of homogeneous space is that they behave in some way at any point. It follows that if we Know how the bornology of bornological group behaves at the identity, we now this bornology everywhere. This observation suggests a certain approach to bornologies a group $G$, that means if bornological set is homogenous. So, it can bornologies every group to bornological group.

On the contrary, a bornological semigroup, even if it has identity, need not be homogenous, then the example below shows that Theorem 3.4 cannot be extended to bornological semigroup.

Example 3.5. Take the bounded unit interval $J=[0,1]$, and put $x y=\max \{x, y\}$ for all $x, y \in J$. Clearly, $J$ with the canonical bornology and this product operation, is a bornological semigroup (with 0 in the role of identity). However, $J$ is not a homogeneous space since no isomorphism of $J$ takes, 0 to $1 / 2$.

We describe here some simple facts on families of bounded sets in bornological groups. We start with the proposition which shows, in bornological groups there is an intimate relationship between the set of the form $H B$, where $B$ is bounded and $H$ any subset.

Proposition 3.6. If $G$ is a left bornological group then, for any bounded subset $B$ of $G$ and any subset $H$ of $G$, the sets $B H$ and $H B$ are bounded.

Proof. Every left translation of $G$ is an isomorphism, by Proposition 3.2. Since $H B=$ $\bigcup_{h \in H} l_{h}(B)$, the conclusion follows.

A similar argument applies in the case when $G$ is a right bornological group.

So, if $G$ is a left (right) bornological group then, for any bounded subset $B$ of $G$ and any subset $H$ of $G$, the sets $B H$ and $H B$ are bounded.

We obtain immediately the following corollary.

Corollary 3.7. Suppose that a subgroup $H$ of a bornological group $G$ contained in a non-empty bounded subset of $G$. Then $H$ is bounded in $G$.

Proof. Let $B$ be a bounded subset of $G$ with $H \subset B$. A left bornological group consists of a group $G$ and a bornology $\beta$ on $G$ such that $\beta$ is stable under hereditary. Then, the set $H \subset B$ is bounded in $G$.

Theorem 3.8. Let $f: G \longrightarrow H$ be a homomorphism, where $\left(G, *, \beta_{G}\right)$ and $\left(H, \circ, \beta_{H}\right)$ are bornological groups. If $f$ is bounded map at the identity element $e_{G}$ of $G$, so $f$ is bounded map on $G$.

Proof. let $g \in G$ be an arbitrary element, and let $B$ be a bounded set containing $g$ in $G$. Since the left translations in $G$ are bounded map, there is a bounded set $B_{1}$ containing identity element $e_{G}$ of $G$ such that

$$
l_{g}\left(B_{1}\right)=g * B_{1} \subset B .
$$

Since $f$ is bounded map at $e_{G}$ of $G$, it follows the existence of a bounded set $B_{2} \subset H$ containing $e_{H}$ such that $B_{2} \subset f\left(B_{1}\right)$. But

$$
l_{g_{1}=f(g)}: G \longrightarrow G
$$


is a bounded mapping, so that the set $f(g) \circ B_{2}$ is a bounded set containing $g_{1}=f(g)$, for which we have

$$
f^{-1}\left(f(g) \circ B_{2}\right) \subset g * f^{-1}\left(B_{2}\right) \subset g * B_{1} \subset B .
$$

Hence $f$ is bounded map at $g$ of $G$, and since $g$ was an arbitrary element in $G, f$ is bounded map on $G$

Next we will discuss embeding of a group.

Definition 3.9. Given that for all $\alpha \in I$ we have $G_{\alpha}=G$, we denote the product $\prod_{\alpha \in I} G_{\alpha}=G \times G \times G \ldots \times G$ as $G^{I}$. We can view $G$ as the set of all maps from $I$ to $G$. Consider the element $g \in G$ as $g=\left(g_{\lambda_{1}}, g_{\lambda_{2}}, g_{\lambda_{3}}, \ldots, g_{\lambda_{i}} \ldots\right)$ we can associate this element with the function $f_{g}: I \longrightarrow G^{I}$ that maps $\lambda_{1}$ to $g_{\lambda_{i}}$ for all $\alpha \in I$

Proposition 3.10. Let $G$ be a bornological group. There exists a bijection from $G$ onto a subset of $G^{G}$. This map is embedding of $G$.

Proof. Define map from $G$ to $G^{G}$ by $\psi_{r}: g \longrightarrow r_{g}$ and $\psi_{l}: g \longrightarrow l_{g}$. In this proof we will use $\psi_{r}$ but the left translation also work. In the beginning, we have to show that $\psi_{r}$ is injective, so assume that $\psi_{r}=\psi_{l}$ for some $x, y \in G$. It follows that $r_{x}=r_{y}$, so $g x=g y$ for all $g \in G$, multiplying by $g^{-1}$ on the left gives us $x=y$ as required. Then $\psi_{r}: G \longrightarrow \psi_{r}(G)$ is a bijection. We call $\psi_{r}$ and $\psi_{l}$ the right and left canonical embedding of $G$ into $G^{G}$.

\section{Conclusion}

This paper deal with study to understand what conditions should be imposed on bornological set to guarantee that the bornological set will turn group into (BG). As well as, the main important results, that is, in every bornological group all right (left) translation are isomorphisms and so bornological group structure is homogeneous. Thus, we solve the problem of which bornological condition on bornological set can give a natural way to bornologies any group onto (BG). Furthermore, In contrast with bornological group, we show bornological semigroup is not homogenous space.

\section{ACKnowledgment}

The research is supported by the Department of Mathematics Universiti Putra Malaysia, Malaysia.

\section{REFERENCES}

[1] F. Bambozzi, On a Generalization of Affinoid Varieties, Ph.D Thesis, Universit degli Studi di Padova, Dipartimento di Matematica, (2013).

[2] F. Bambozzi, Closed Graph Theorems for Bornological Spaces, arXiv preprint arXiv:1508.01563, (2015).

[3] H. Hogbe-Nlend, Bornologies and Functional Analysis, North - Holland Publishing Company, Netherlands, (1977).

[4] A. N. Imran and I. S. Rakhimov, On Bornological semi groups, IEEE proceeding in ICREM7, (2015).

[5] A. N. Imran and I. S. Rakhimov, On Semi Rings Bornologies, ATP proceeding in ICMSS, (2016).

[6] A. Lechicki, S. Levi and A. Spakowski, Bornological Convergences, J. Math. Anal. Appl, 297, 2, 751-770, (2004).

[7] D. P. Pompo Jr., Bornological Groups, Indian J. Math, 54, 225-258, (2012). 
(C) 2017 by the authors; licensee Preprints, Basel, Switzerland. This article is an open access article distributed under the terms and conditions of the Creative Commons Attribution (CC BY) license http://creativecommons.org/licenses/by/4.0/).

A.N. Imran ${ }^{1}$, Department of Mathematics, FS, Universiti Putra Malaysia, Malaysia and Dept. of Math., Faculty of Sciences, University Dyala, Baqubah, Iraq

I.S. Rakhimov 2 , Department of Mathematics, FS and Institute for Mathematical Research (InSPem), Universiti Putra Malaysia, 43400 Serdang, Selangor Malaysia E-mail address: ${ }^{1}$ anwarnooraldeen@yahoo.com $\quad{ }^{2}$ risamiddin@mail.ru 